Title:

Author(s):

Submitted to:

\title{
Multifunctional Self-Assembled Monolayers
}

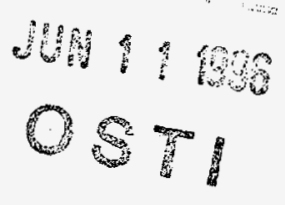

Thomas Zawodzinski, MST-11

Georg Bar

John Ferrais, University of Texas, Dallas

Shai Rubin

Francisco Uribe, MST-11

DOE Office of Scientific and Technical

Information (OSTI)

\section{Los Alamos}

NATIONAL LABORATORY

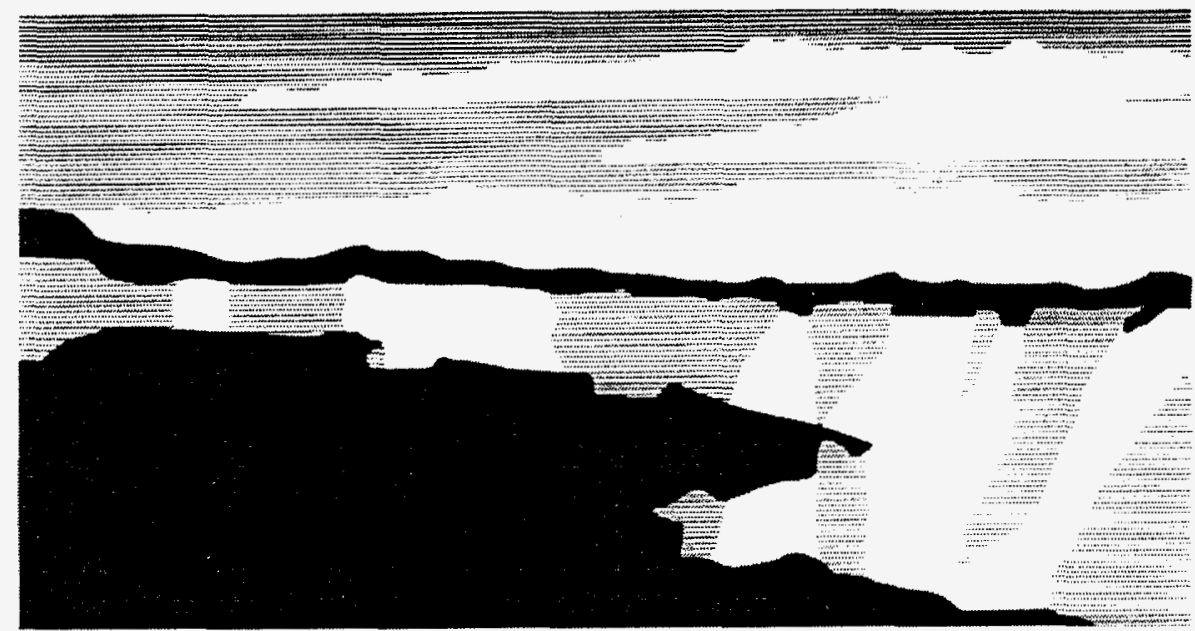

Los Alamos National Laboratory, an affirmative action/equal opportunity employer, is operated by the University of Catifornia for the U.S. Department of Energy under contract W-7405-ENG-36. By acceptance of this article, the publisher recognizes that the U.S. Government retains a nonexclusive, royaltyfree license to publish or reproduce the published form of this contribution, or to allow others to do so, for U.S. Govemment purposes. The Los Alamos National Laboratory requests that the publistyer ichntity ghis articje as work periormed under the auspices of the U.S. Department of Energy.

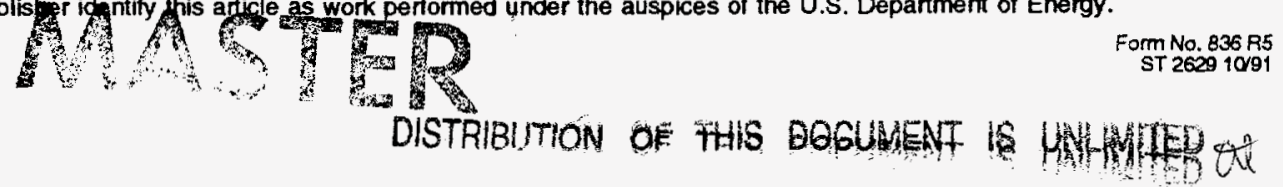




\section{DISCLAIMER}

This report was prepared as an account of work sponsored by an agency of the United States Government. Neither the United States Government nor any agency thereof, nor any of their employees, makes any warranty, express or implied, or assumes any legal liability or responsibility for the accuracy, completeness, or usefulness of any information, apparatus, product, or process disclosed, or represents that its use would not infringe privately owned rights. Reference herein to any specific commercial product, process, or service by trade name, trademark, manufacturer, or otherwise does not necessarily constitute or imply its endorsement, recommendation, or favoring by the United States Government or any agency thereof. The views and opinions of authors expressed herein do not necessarily state or reflect those of the United States Government or any agency thereof. 


\section{DISCLAIMER}

Portions of this document may be illegible in electronic image products. Images are produced from the best available original document. 


\title{
Multifunctional Self-Assembled Monolayers
}

\author{
Thomas Zawodzinski*, Georg Bar, John Ferrais (University of Texas, Dallas), \\ Shai Rubin, and Francisco Uribe
}

\begin{abstract}
This is the final report of a three-year, Laboratory-Directed Research and Development (LDRD) project at the Los Alamos National Laboratory (LANL). The specific goals of this research project were threefold: (1) to develop multifunctional self-assembled monolayers, (2) to understand the role of monolayer structure on the functioning of such systems, and (3) to apply this knowledge to the development of electrochemical enzyme sensors. An array of molecules that can be used to attach electrochemically active biomolecules to gold surfaces has been synthesized. Several members of a class of electroactive compounds have been characterized and the factors controlling surface modification are beginning to be characterized. Enzymes have been attached to self-assembled molecules arranged on the gold surface, a critical step toward the ultimate goal of this project. Several alternative enzyme attachment strategies to achieve robust enzyme-modified surfaces have been explored. Several means of juxtaposing enzymes and mediators, electroactive compounds through which the enzyme can exchange electrons with the electrode surface, have also been investigated. Finally, the development of sensitive biosensors based on films loaded with nanoscale-supported gold particles that have been surface modified with the self-assembled enzyme and mediator have been explored.
\end{abstract}

\section{Background and Research Objectives}

The preparation of organized microstructures on electrode surfaces is of interest in various areas, including molecular electronics, photochemical conversion, electrocatalysis, and chemical sensor development. A large body of work exists in which Langmuir-Blodgett bilayer films have been transferred from the air/water interface onto solid electrodes using mechanical pressure. Such structures are inherently fragile. Interaction of the bilayer with the surface is non-specific. These properties are to be contrasted with self-assembled monolayers

*Principal investigator, e-mail: zawod@lanl.gov 
(SAMs) derived from materials adsorbed on or attached to a surface via a strong, chemicallyspecific interaction.

Self-assembled monolayers have the virtue of providing dense, stable assemblies of molecules that can be selectively grafted to a wide spectrum of chemically active groups. They can also provide sufficient electron tunneling to allow electrochemical control of reactions with these active groups. Enzymes are particulariy attractive active groups because they provide unparalleled chemical selectivity. By coupling this selectivity with an electrochemical process, specific reactions can be sensed or controlled electronically. This electronic control provides the opportunity to interface a chemically engineered surface with electronics. The most common type of monolayer constructed on gold electrodes is based on the strong interaction of thiols with gold surfaces and is thus quite stable.

The objective of this project was to establish SAMs as a tool for controlling enzymatic electrochemical reactions. The capability to tailor a surface for specific electrochemical reactions is a potentially important element in molecular electronics and could have a strong impact on the development of sensors for a variety of substances.

The overall goal of this project was to develop methods of juxtaposing, in selfassembled layers, redox active enzymes and other complexes and to study their interactions with each other. This is a specific example of a multi-step, electron-transfer catalytic complex on an electrode surface, with lateral electron transfers between adjacent elements introduced into the SAM as the key feature. The tasks can be divided into several areas: (1) synthesis and self-assembly of the desired functionalized alkanethiol molecules; (2) characterization of the properties of each of the individual components, particularly their electron transfer properties; (3) attaching enzymes to functionalized alkanethiol modified surfaces; (4) characterization of mixed monolayers of various components; (5) demonstration of interaction amongst all components; and (6) understanding the role of monolayer structure on the functioning of such systems leading to applications of the chemistry such as to electrochemical enzyme sensors.

\section{Importance to LANL's Science and Technology Base and National R\&D Needs}

We have developed a set of chemical methods of wide applicability by using simple yet versatile chemistry that enables the development of complex systems from simple building blocks. For example, the work carried out in this project represents an exciting and novel approach to the development of chemical sensors. Chemical sensors, particularly for environmental monitoring, are of increasing importance to both the DOE and the Laboratory. The sensor concepts contained in this project are adaptable to environmental problems, 
including gas and ground water sensing applications. In addition, the model application studied here extends the Laboratory's capability in biomedical technology. Finally, the understanding and control of surface chemical architecture are valuable for molecular electronics applications.

\section{Scientific Approach and Resuits to Date}

This project involves activities in several areas including substrate preparation, monolayer self-assembly, synthetic methods for introducing enzymes and electrochemically active moieties into surface films and measurements of electrochemical response of mixed monolayers of ferrocenylalkanethiols and alkanethiols. Substrate preparation and monolayer self assembly have been employed extensively as an underlying basis for continuing work. Electrochemical studies of monolayers containing ferrocenylalkanethiols as one component have been conducted. However, detailed studies emerging from other groups have provided the necessary background to our work and thus we lowered the emphasis on this facet of the project.

One general aspect of our use of self-assembly methods that is fundamental to the application addressed in this work as well as in development of more wide-ranging applications of these methods is the preparation of a "molecular tool kit" of molecules functionalized such that they allow a wide range of post-reactions for building up of complex surface architectures for functional applications. Examples of the "hooks" to which we attach functional units include alkanethiols terminating in amine, aldehyde or carboxylate groups. We have such molecules in hand.

\subsection{Enzyme/Mediator Electrocatalytic Systems}

The use of an alkanethiol-based, self-assembled mixed monolayer as an electronic relay system effecting mediated electron transfer between immobilized glucose oxidase (GOx) and a gold electrode was studied in depth. Initially, we compared the behavior of mixed monolayers of various compositions of 16-ferrocenylhexadecanenthiol (16FAT, the number refers to chain length) and aminoalkanethiol (2AmAT), to which GOx is attached, as biosensors for glucose. Our experiments show that the amperometric response of such electrodes in the presence of glucose in solution depends on the mole ratio between the 16FAT molecules and the attached protein molecules in the monolayer. As shown in Figure 1a, the most sensitive system is a mixed monolayer that contains $7 \%$ 16FAT. For higher 16FAT concentrations, both a catalytic response and a wave corresponding to reversible $16 \mathrm{FAT}$ voltammetry are observed in the presence of glucose. This suggests that there are separate domains of $16 \mathrm{FAT}$ and of $2 \mathrm{AmAT}$ 
in such a mixed monolayer. When the mixed monolayer contains more than $7 \%$ of $16 \mathrm{FAT}$, some 16FAT molecuies cannot "feel" the GOx, and do not function as electron transfer relays.

There are two interesting points to explain regarding the data which could provide important insight into the factors underlying function of this catalytic assembly: (1) in the absence of glucose, there no electrochemical response of the 16FAT population that interacts with the enzyme and (2) the question of the source of the large difference in the redox potential (almost $-400 \mathrm{mV}$ in $E_{p} / 2$ value or -150 to $-320 \mathrm{mV}$ in $E_{p}$ values) between the two populations and among the three different loadings that are sensitive towards glucose The reaction scheme for the catalytic process occurring in the presence of glucose is:

$$
\begin{gathered}
\mathrm{Fc}=\mathrm{Fc}^{+}+\mathrm{e}^{-} \\
\mathrm{Fc}^{+}+\mathrm{GOx}_{\mathrm{red}}=\mathrm{Fc}+\mathrm{GOx}_{\mathrm{OX}} \\
\text { glucose }+\mathrm{GOx}_{\mathrm{OX}}=\mathrm{GOx}_{\mathrm{red}}+\text { gluconolactone }
\end{gathered}
$$

where $\mathrm{Fc}$ is ferrocene, $\mathrm{Fc}^{+}$is ferricenium ion, GOx red is the reduced form of GOx (GOx$\mathrm{FADH}_{2}$ ), $\mathrm{GOx}_{\mathrm{Ox}}$ is the oxidized form of GOx (GOx-FAD).

Two possible explanations for the observed shift are (1) a kinetic explanation in which the electrochemically sensed reaction 1 is driven to the left since all $\mathrm{Fc}^{+}$is rapidly consumed by reaction 2 and (2) an environmental explanation in which the ferrocene voltammetry is affected by the hydrophobicity of the surrounding environment or has its structure perturbed by interaction with the enzyme in the monolayer. It is hard to believe the environmental explanations since (1) the enzyme aggregate environment is more hydrophobic than buffer solution, leading to a shift of opposite sign; (2) only a dramatic change of the environment can cause such a big shift in the formal potential; and (3) this explanation does not directly explain that, in the absence of glucose, the absence of a redox peak from the relay Fc.

The existence of these domains was also characterized by studying the solution voltammetry of $\mathrm{Ru}\left(\mathrm{NH}_{3}\right) 6^{3+}$ at electrodes with various proportions of $16 \mathrm{FAT}$ and $2 \mathrm{AmAT}$. A typical family of curves is shown in Figure 2. As the amount of 2AmAT in the layer was increased, the current-voltage characteristic varied from a tunneling current, typical of electron transfer through a well-insulated layer, through a steady state curve, typical of an array of microelectrodes, to a normal cyclic voltammogram, typical of semi-infinite linear diffusion. This behavior suggests that the 2AmAT forms islands of low order, allowing penetration of the redox probe close to the surface, another indicator of domain formation, while the FAT portions of the electrode are well-packed and do not allow efficient electron transfer through them.

Mixed monolayers containing AmATs and FATs of other combinations of chain lengths were also prepared and characterized by electrochemistry, X-ray photoelectron spectroscopy (XPS), contact angle and atomic force microscopy (AFM). These mixed monolayers were also 
modified by GOx attachment to the amine. The relative efficiency as a biosensor of each chainlength combination of FAT and AmAT was examined by measuring its response toward different glucose concentrations. In light of this comparison, we considered the critical factors for efficient electron transfer between the ferrocene mediator and the GOx redox active site immobilized as part of the surface-confined system. The electrochemical results for various chain length combinations are shown in Figure 1. Interestingly, the catalytic behavior of these systems is worse when the chain lengths are similar in length, improving with dissimilar chain lengths. Our explanation for this effect is detailed below for the various combinations of alkane chain lengths used.

\section{12 FAT:6AmAT}

Voltammetry is shown in Figurg $1 \mathrm{~b}$. The chain length of the ferrocene spacer and the enzyme spacer are equal. We therefore expect that the FAT has limited ability to move. The enzyme is deposited on the surface of the mixed monolayer. The 12FAT:6AmAT system is a very rigid system. The enzyme situated on the surface of the monolayer is analogous to an enzyme attached directly to an electrode surface. In the latter case, achieving electronic communication between GOx and an electrode is not straightforward [1].

\section{8 FAT:10AmAT}

In this system, the mediator is buried in the mixed monolayer and the enzyme spacer has some mobility. Figure 1c shows the amperometric response of electrode surfaces modified with a variety of proportions of the two components. In this case, the sensitivity is better than the sensitivity of the 12FAT:6AmAT system but it is still only approximately $40 \%$ (for the optimal composition, $19 \%$ loading) of the sensitivity of 16FAT:2AmAT system. Because the enzyme has to penetrate into the mixed monolayer to react with the mediator molecule, the amperometric response of this system is somewhat surprising if we think of it as a mixed monolayer with random distribution of the two components. A reasonable explanation for the relative sensitivity of the 8FAT:10AmAT system is that the 8FAT domains are sufficiently large (approximately $100 \AA^{2}$ ) that the enzyme can extend into the mediator domain thanks to the mobility of its spacer.

\section{6 FAT:10AmAT}

This case tests the hypothesis formed from the 8FAT:10AmAT case. Figure 1d shows the amperometric response of the system 6FAT:10AmAT modified by GOx. It is clear that in this case the mobility of the enzyme spacer is even larger then in the 8FAT:10AmAT case because of the huge length difference between the two spacers.

We conclude that the critical factor controlling the electron transfer step in these systems is ability of the protein to approach the electron mediator. When at least one of the components (the FAT or the enzyme) is mobile, the electron transfer process is possible. However, when 
the system is rigid, as in the case of the 12FAT:6AmAT mixed monolayer, the efficiency of the electron transfer between the enzyme and the mediator is poor. Though it is hard to imagine that the large enzyme molecule can penetrate through the FAT region to reduce the distance between the active site and the mediator when the alkane chain of the FAT is short, it is nonetheless possible that the enzyme can interact with the mediator on the surface in a more favorable conformation for the electron transfer process in a flexible system than in the case when the system is rigid. Thus, we have developed an understanding of structural requirements for optimal performance of mixed monolayer electrocatalytic systems as glucose sensing electrodes.

\subsection{Patterning: Lithography, Stamping}

Our discovery of domains underlying the behavior of the enzyme/mediator systems led us to begin to prepare artificial domains via patterning of surfaces. Several methods for this exist in the literature. We prepared appropriate masks for lithography and proceeded to prepare systems with patchwork patterns and having alternating squares of FATs and ATs.

Unfortunately, the ferrocene moiety tended not to survive the lithographic process, as indicated by the observed electrochemical response of the lithographically patterned samples. Recently, using slightly altered conditions, successful patterning has been achieved.

A second approach we took to the preparation of controlled surface architectures was the adaptation of stamping methods, as described by Whitesides, et al. [2]. The stamping process is carried out in several steps: (1) preparation of a polymeric stamp with the desired pattern; (2) immersion of the stamp in the appropriate functionalized alkanethiol solution; (3) contact of the loaded stamp with the gold surface, thus effecting transfer of functionalized alkanethiol molecules; and (4) liftoff of the stamped sample and immersion into a solution of second component. A key issue in this method is the interdiffusion of the two components at the boundaries. Quite sharp edges could be achieved as shown in scanning electron microscopy (SEM) images (Figure 3).

An immediate need was characterization of the patterned structures. This was accomplished by scanning probe methods. An interesting synergy emerged between our attempts to pattern surfaces with SAMs of different functionality and the development of scanning force microscopy (SFM) methods for imaging this patterning. The patterned SAMs are excellent model systems for understanding the forces contributing to observed frictional interactions between probe tip and sample. Because of our molecular-level control over elements of the self-assembled pattern, the packing, the hydrophobicity, and the charge of the exposed end of the self-assembled monolayer, we can simultaneously obtain the information we want about patterns and extend achievable resolution of scanning probe methods. 
We prepared mixed-pattern SAMs of alkanethiols terminated by different chemical functionalities (e.g., $-\mathrm{CH}_{3},-\mathrm{NH}_{2},-\mathrm{OH},-\mathrm{COOH}$ ). Binding of intact molecules to the surface was verified using electrochemical and surface-sensitive spectroscopic techniques, i.e., cyclic voltammetry, XPS and scanning Auger electron spectroscopy (AES). We used scanning force microscopy techniques (AFM/LFM) to image the patterned surfaces and to measure the frictional and adhesive forces between the probe tip and regions of SAMs terminated by different chemical functionalities. Lateral force microscopy (LFM), which probes the in-plane frictional interaction between the probe tip and the sample, provides excellent imaging of the patterned SAMs with high resolution. In conjunction with AES, it is clear that the LFM image contrast between the scanning tip and the alkanethiols depends on the terminal groups. For a silicon tip, the friction force was in general found to be higher over the more hydrophilic regions. The friction also depends on the hydrocarbon chain length and the applied load. The adhesion forces between the different arrays of the SAMs and the unmodified and modified probe tip were investigated by measuring force-versus-displacement curves. Qualitatively, the trend of the adhesive forces between hydrophobic, hydrophilic and acid-base groups is as expected based on the interaction between those groups.

A particularly interesting study illustrates the power of this approach. A patterned sample of hexadecanethiol (16AT) and 12FAT was characterized by SEM, AFM, Auger spectroscopy, electrochemistry and contact angle measurements. This work represents the first report of images, obtained with an unmodified tip, with good contrast between regions of similar hydrophobicity. The high-quality SFM images, shown in Figure 4, obtained for this patterned sample, in which the two components of the pattern are fairly similar in hydrophobicity, suggests that the driving force for the recognition between the SFM tip and chemically different regions arises not only from hydrophobic/hydrophilic or electrostatic interactions but also from basic materials features.

\subsection{Colloids}

We have described a novel approach for gold- and silver-colloid-monolayer formation on different silicon oxide surfaces such as glass, silicon and indium-doped tin oxide (ITO). The preparation method is simple and yields monolayers with easily controlled spacing within the monolayer ,but without aggregation of metal particles. The colloid monolayers are prepared in two steps: (1) modification of the substrates with star burst dendrimers; (2) noblemetal-colloid deposition onto the dendrimer layer. The structure and properties of the resulting particle arrays have been studied by various methods. Data from XPS measurements show that the dendrimers spontaneously adsorb to various silicon oxide surfaces. The thickness of the dendrimer overlayer has been calculated to lie in the $d=14-25 \AA$ range. The SEM and 
AFM data show that the colloids spontaneously form continuous films on the dendrimermodified surfaces. The noble-metal particles are well isolated, confined to a single layer and not aggregated on the surface. The interparticle spacing (74-829 nm) and surface coverage can be controlled over a wide range by colloid size, colloid concentration and immersion time. Ultraviolet-visible spectroscopic data show that the microstructure directly controls the optical properties of the layer. We demonstrated that the prepared substrates provide a useful platform for surface-enhanced Raman spectroscopy (SERS) studies of materials adsorbed on the metal particles. These colloidal samples can be used to prepare highsurface-area, gold-composite electrodes for applications of the SAMs.

\subsection{Other Applications of SAMs}

We have made preliminary forays into extending the utility of the SAMs that can be prepared. These applications demonstrate the wide range of applicability of our tool kit of molecules.

\section{Precoats for Modifying Electronic Properties of Metals}

In collaboration with other LANL investigators, we have modified gold and silver surfaces with alkanethiols terminating in $-\mathrm{NH} 2,-\mathrm{COOH}$, or $-\mathrm{H}$ and demonstrated shifts in the electroabsorption curves consistent with the signs of the dipoles of the various molecules. This indicates that the dipole moment of the alkanethiol alters the electronic properties of the metal surface. This effect is potentially useful in constructing interfaces with matched electronic properties for electron or hole injection.

2. Azobenzene: Reading/Writing/Erasing

We prepared an azobenzene-terminated alkanethiol. The object of this work was to develop a combined electrochemical/optical read-write-erase device for memory storage based on the ability to photoswitch between cis- and trans-forms of azobenzene. The cis- and trans- forms have different electrochemical properties.

\section{Light Harvesting Devices}

We are preparing a donor/acceptor/sensitizer triad which can be self-assembled using our methods. Studies of charge separation in such devices, corresponding to lateral electron transfers, when combined with structural control, achievable using various chain lengths for example, could yield insight into optimal configurations for light harvesting.

\section{References}

1. Heller, A. J. Phys. Chem, 1992, 96, 3579.

2. Kumar, A.; Whitesides, G. M. Appl. Phys. Lett. 1993, 63, 2002. 


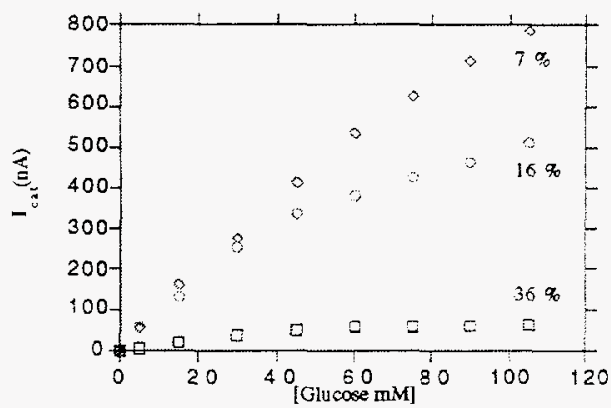

16FAT:aminoethanethiol

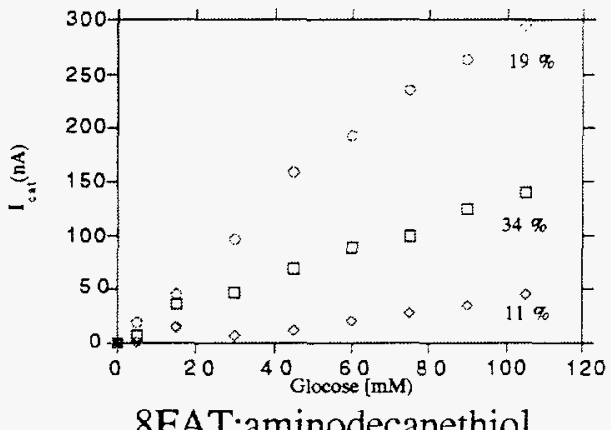

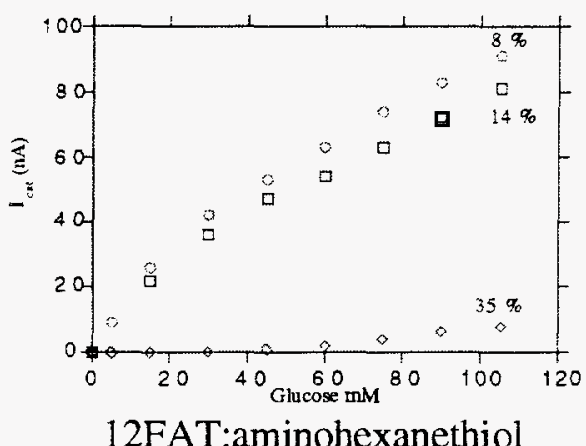

12FAT:aminohexanethiol

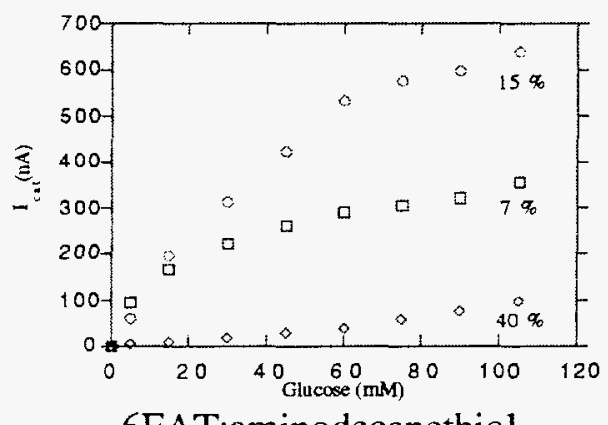

6FAT:aminodecanethiol

Figure 1. The amperometric response of the different systems towards different concentrations of glucose: (a) 16 FAT:aminoethanethiol modified by GOx, (b) 12 FAT:aminohexanethiol modified by GOx, (c) 8 FAT:aminodecanethiol modified by GOx, (d) 6 FAT:aminodecanethiol modified by GOx. 

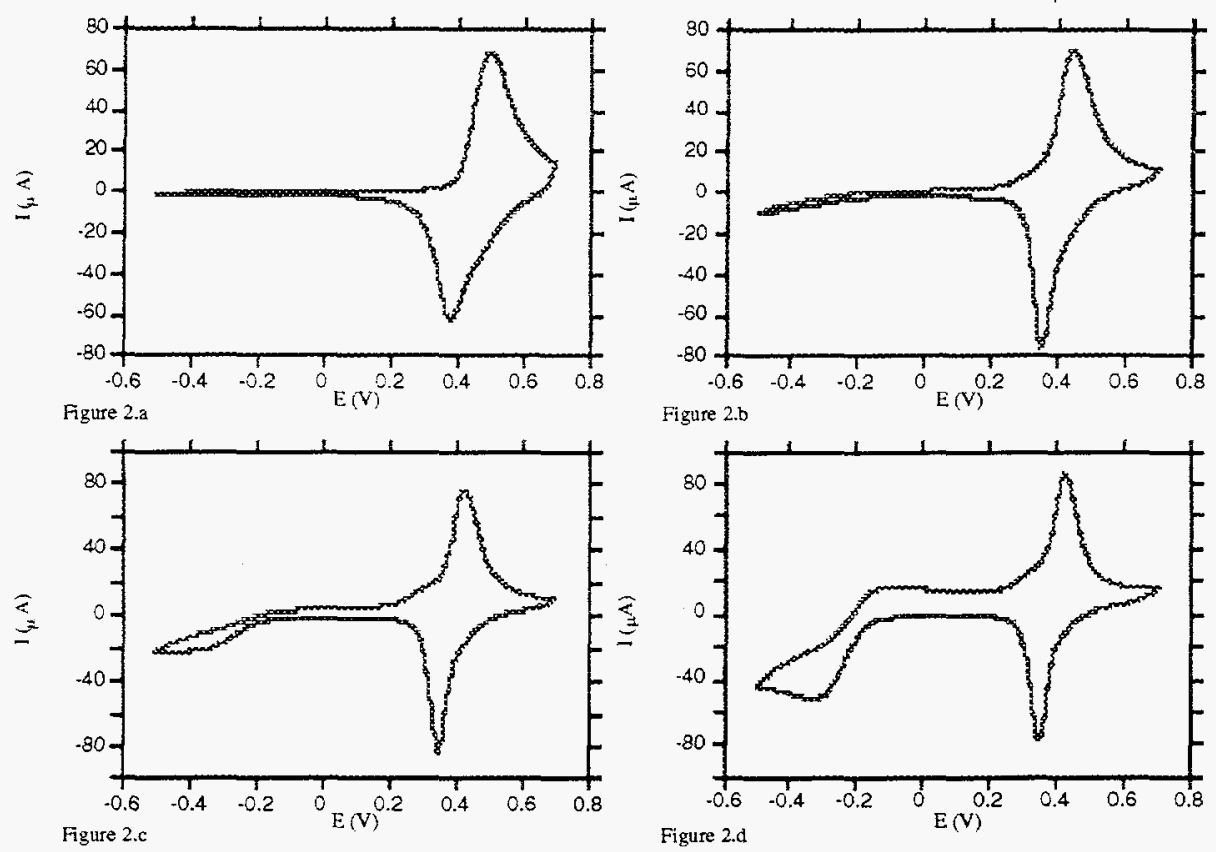

Figure $2 . \mathrm{b}$
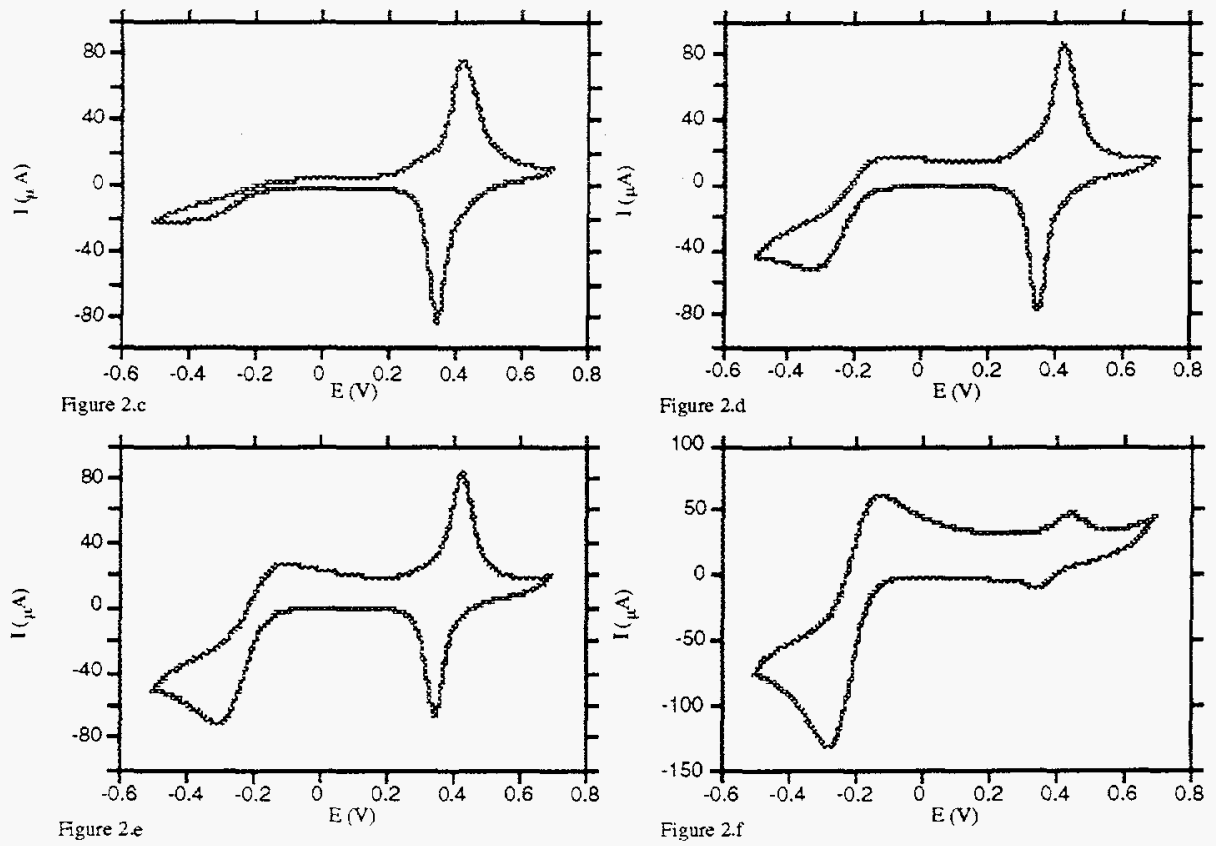

Figure 2: The electrochemical response of solutions of $\mathrm{Ru}\left(\mathrm{NH}_{3}\right) 6^{3+}$ at electrodes coated with 16FAT:aminoethanethiol mixed monolayers of various compositions. (a) Monolayer composed only from 16FAT; (b) $53 \%$ loading of $16 \mathrm{FAT}$; (c) $49 \%$ loading of $16 \mathrm{FAT}$; (d) $43 \%$ loading of $16 \mathrm{FAT}$; (e) $36 \%$ loading of $16 \mathrm{FAT}$; (f) $8 \%$ loading of $16 \mathrm{FAT}$. 


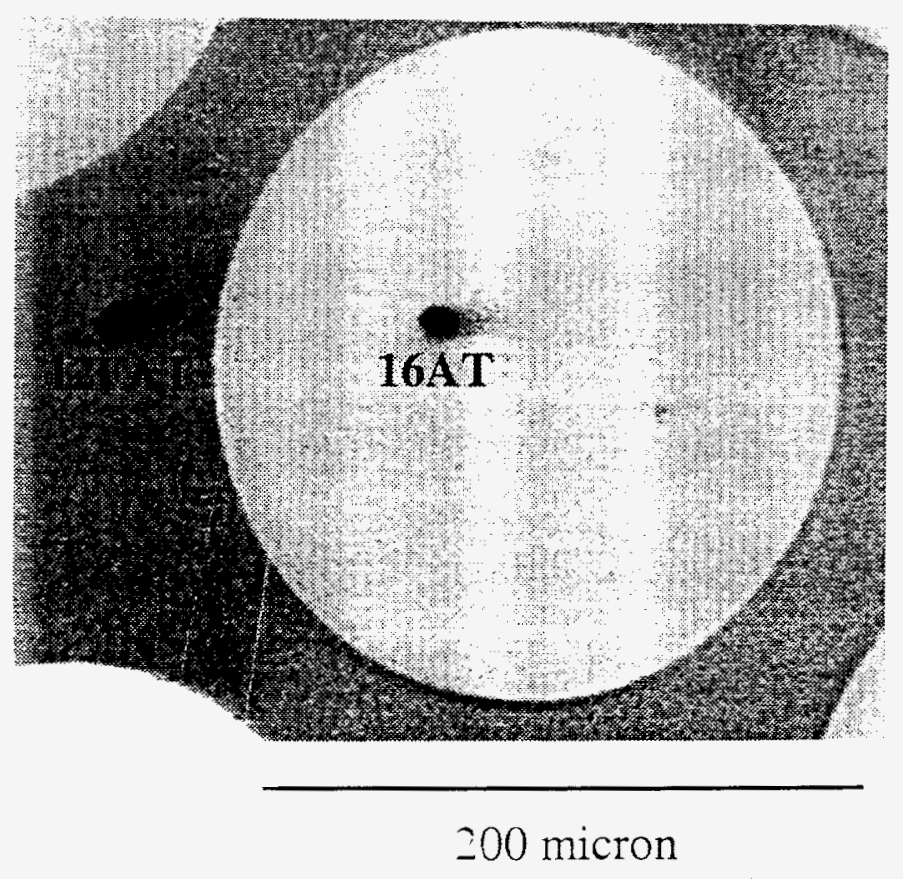

Figure 3. SEM image of stamped pattern. Inside 200 micron disk is 16AT: outside is $12 \mathrm{FAT}$.

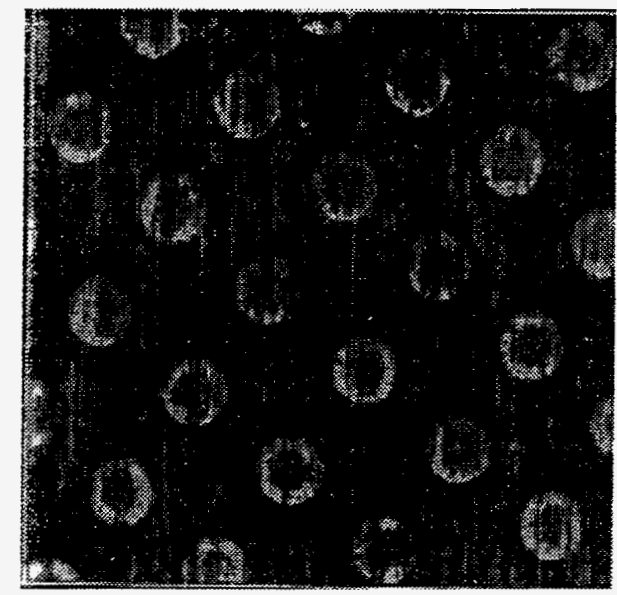

Figure 4. LFM image of stamped pattern. Inside 5 micron disks is 12FAT: outside is 16AT. 